\title{
Estudo sobre câncer gástrico, seus fatores de risco e prognósticos: uma revisão integrativa
}

\author{
Study on gastric cancer, its risk factors and prognosis: an integrative review
}

Estudio sobre el cáncer gástrico, sus factores de riesgo y pronóstico: una revisión integradora

Larissa Sant'Ana Brum ${ }^{1 *}$, Cristina Maria Monteiro Dantas ${ }^{1}$, Manuella da Silva Machado ${ }^{1}$, Mariana Saracino de Almeida ${ }^{1}$, Isabela Costa Linhares ${ }^{1}$, Adriana Rodrigues Ferraz ${ }^{1}$.

\section{RESUMO}

Objetivo: Verificar através de uma revisão integrativa os fatores de risco e os fatores prognósticos para o câncer gástrico. Métodos: $O$ presente trabalho trata-se de uma revisão integrativa de literatura embasado em evidências, cuja elaboração deu-se por meio das seguintes etapas: identificar um tema e selecionar a questão de pesquisa, buscar os artigos nas bases de dados digitais e estabelecer critérios de inclusão e exclusão com posterior análise e discussão dos resultados obtidos nos periódicos. Resultados: São descritos como fatores de risco: a infecção pelo Helicobacter pylori (H. Pylori) em que precisa estar associada a hábitos de vida (tabagismo, etilismo) e a predisposição genética, sendo o que determina o aparecimento de lesões consideradas precursoras do adenocarcinoma; fatores dietéticos; baixa escolaridade; renda salarial; idade e IMC. Em relação ao prognóstico: o estadiamento do tumor torna-se determinante para a escolha do melhor tratamento, o estado nutricional do paciente, a presença de comorbidades e o avançar da idade do paciente são relatados como os aspectos mais relevantes. Considerações finais: Confirma-se a importância da atenção aos fatores de risco e do diagnóstico precoce, a fim de oportunizar melhor qualidade de vida e um melhor prognóstico para os pacientes.

Palavras-chave: Câncer, Gástrico, Câncer gástrico, Prognóstico, Fatores de risco.

\begin{abstract}
Objective: Verify through an integrative review the risk factors and prognostic factors for gastric cancer. Methods: The present work is an integrative literature review based on evidence, whose elaboration took place through the following steps: identify a theme and select the research question, search the articles in digital databases and establish inclusion criteria and exclusion with further analysis and discussion of the results obtained in the journals. Results: The following risk factors are described: infection by Helicobacter pylori $(\mathrm{H}$. pylori), which needs to be associated with lifestyle habits (smoking, alcoholism) and genetic predisposition, which determines the appearance of lesions considered precursors of adenocarcinoma; dietary factors; low education level; wage income; age and BMI. Regarding the prognosis: tumor staging is crucial for choosing the best treatment, the patient's nutritional status, the presence of comorbidities and advancing patient age are reported as the most relevant aspects. Conclusion: The importance of attention to risk factors and early diagnosis is confirmed, in order to provide better quality of life and a better prognosis for patients.
\end{abstract}

Key words: Cancer, Gastric, Gastric cancer, Prognosis, Risk factors.

${ }^{1}$ Universidade de Vassouras (UV), Vassouras - RJ.

*E-mail: Isbrum-2310@hotmail.com

SUBMETIDO EM: 11/2021

ACEITO EM: 11/2021

PUBLICADO EM: 11/2021 


\section{RESUMEN}

Objetivo: Verificar mediante una revisión integradora los factores de riesgo y factores pronósticos del cáncer gástrico. Métodos: El presente trabajo es una revisión integradora de la literatura basada en la evidencia, cuya elaboración se llevó a cabo a través de los siguientes pasos: identificar un tema y seleccionar la pregunta de investigación, buscar los artículos en bases de datos digitales y establecer criterios de inclusión y exclusión con mayor análisis y discusión de los resultados. obtenido en las revistas. Resultados: Se describen los siguientes factores de riesgo: infección por Helicobacter pylori (H. pylori), que debe estar asociada a hábitos de vida (tabaquismo, alcoholismo) y predisposición genética, que determina la aparición de lesiones consideradas precursoras del adenocarcinoma; factores dietéticos; bajo nivel educativo; ingresos salariales; edad e IMC. En cuanto al pronóstico: la estadificación tumoral es crucial para elegir el mejor tratamiento, el estado nutricional del paciente, la presencia de comorbilidades y el avance de la edad del paciente se reportan como aspectos más relevantes. Conclusión: Se confirma la importancia de la atención a los factores de riesgo y el diagnóstico precoz, con el fin de brindar una mejor calidad de vida y un mejor pronóstico a los pacientes.

Palabras clave: Cáncer, Gástrico, Cáncer gástrico, Pronóstico, Factores de riesgo.

\section{INTRODUÇÃO}

O Câncer Gástrico (CG) constitui uma das principais causas de letalidade por neoplasia em todo o mundo. Apesar de observar-se a nível mundial uma redução na sua incidência nas últimas décadas, o número absoluto de casos por ano vem apresentando aumento (LOPES AC, et al., 2020). Aproximadamente 22.220 pacientes são diagnosticados anualmente nos Estados Unidos e dentre esses 10.990 provavelmente evoluirão para óbito (LOPES AC, et al., 2020).

No ano de 2015, somente no Brasil, foi responsável por 14.264 casos, sendo 9.132 homens e 5.132 mulheres. Estima-se para os anos de 2020 a 2022, 13.360 casos novos em homens e 7.870 em mulheres no Brasil (SANTOS GRAC, et al, 2020).

O número de brasileiros com câncer gástrico torna $C G$ o sexto tumor maligno entre mulheres e quarto entre os homens (SILVA VCS e FELÍCIO DC, 2016). A idade média de acometimento está entre 50-70 anos de idade, sendo, no Brasil, uma das três primeiras causas de morte por câncer (BESSA RA, et al., 2019). Entre as pessoas com baixo nível socioeconômico, a incidência e mortalidade do câncer gástrico são três vezes maiores do que as de pessoas com alto nível socioeconômico (SILVA VCS e FELícIO DC, 2016).

Através de alterações na mucosa gástrica sob ação de fatores ambientais, endócrinos e imunológicos que induzem a modificações das células normais por células neoplásicas, a patogênse do CG é manisfestada (LOPES AC, et al, 2020). O câncer gástrico apresenta tipos histológicos distintos como: adenocarcinoma, linfomas, tumores carcinóides, tumores estromais Gastrointestinais (GIST), liposarcomas e leiomiossarcoma (OLIVEIRA KSM, et al, 2016).

O adenocarcinoma representa mais de $90 \%$ dos casos e subdivide-se em dois tipos morfológicos distintos: intestinal e difuso. Esses dois subtipos guardam diferenças epidemiológicas, etiológicas, patogênicas e prognósticas (ZILBERSTEIN B, et al., 2013).

O tipo intestinal, de maior prevalência, ocorre na mucosa gástrica (a camada de revestimento interna), com características de cânceres ou tumores malignos, surgem na forma de irregulares pequenas lesões com ulcerações (rompimento do tecido mucoso), em que podemos ver na sua carcinogênese uma sequência de etapas de mudança na mucosa: gastrite superficial; gastrite crônica atrófica; metaplasia intestinal; displasia; carcinoma (GONÇALVES S, et al., 2020).

Vários fatores de risco intrínsecos (genéticos) e extrínsecos, também denominados de fatores ambientais são relatados pela literatura. Como principais fatores ambientais identificamos: infecção pelo Helicobacter pylori (H.pylori); alto consumo de sal e alimentos conservados no sal; exposição a compostos $\mathrm{N}$-nitrosos 
(contidos em alimentos defumados, queijos e carnes curadas, tabaco etc.) tabagismo; gastrectomia parcial especialmente do tipo Billroth II; obesidade e baixas condições socioeconômicas. O componente genético é responsável por cerca de 10\% dos adenocarcinomas (JÚNIOR AMF, et al., 2019).

As manifestações clínicas de pacientes com câncer gástrico geralmente têm as seguintes características: Perda de peso rápida e expressiva, indigestão, dor local, náuseas, vômitos, flatulência, saciedade prematura. Esses sintomas podem ser facilmente confundidos com os sintomas de gastrite ou úlcera gástrica o que muitas vezes contribui para o retardo no diagnóstico (DIAS AA, et al., 2016).

A relevância desse tema deve-se ao crescimento do número de casos no Brasil e em todo o mundo, configurando, principalmente nos países subdesenvolvidos, um grave problema de saúde pública, com perspectivas de aumento da mortalidade por essa patologia (SOUZA LT, et al., 2017).

Objetivou-se, portanto, verificar através de uma revisão integrativa os fatores de risco e os fatores prognósticos para o câncer gástrico.

\section{MÉTODOS}

Este artigo trata-se uma revisão integrativa de literatura baseada em evidências, cujo objetivo é a construção de conhecimento fundamentado e uniforme para a realização de uma prática médica de qualidade. Ele se propõe a responder à seguinte pergunta: "Quais são os principais fatores de risco para o aparecimento do câncer gástrico e seu possível prognóstico?".

As etapas para construir essa revisão foram: identificar o tema e selecionar a questão de pesquisa, buscar os artigos nas bases de dados digitais, análise dos artigos com aplicação de critérios para inclusão e exclusão de estudos e discussão dos resultados apresentados pelos periódicos.

Foram buscados artigos publicados no período de 2016 a 2021, nas bases de dados PubMed, Scientifc Eletronic Library Online (Scielo) e Google Acadêmico. Utilizando os seguintes descritores: câncer, gástrico e fatores de risco no PubMed e no Scielo em inglês com o operador "AND" e no Google Acadêmico foram utilizados: câncer gástrico e prognóstico com o operador "AND".

$\mathrm{Na}$ base de dados PubMed foram encontrados no total 13667 artigos, filtrando os achados para o período de tempo 2016 a 2021, sendo encontrados 4438 artigos, filtrado para os estudos observacionais, sendo encontrado 113 e para os textos completos e gratuitos, sendo encontrados 74 .

No Scielo foram encontrados 71 artigos, após a aplicação do filtro no período 2016 a 2021 foram encontrados 9 artigos. No Google Acadêmico as buscas resultaram em 11100 artigos, após aplicação do filtro no período 2016 a 2021 foram encontrados 3700 .

De todas as publicações encontradas nas três bases de dados pesquisadas, foram excluídas as duplicadas, além disso, estabeleceu-se critérios de inclusão e exclusão, e após a aplicação dos mesmos, apenas 12 artigos foram utilizados.

Dentre os critérios de inclusão utilizados temos: estudos que respondem à questão norteadora. A respeito dos critérios de exclusão temos: duplicação entre as bases e artigos fora do tema abordado. Ademais, todas as referências dos 12 periódicos selecionados foram averiguadas por completo, a fim de ampliar o arcabouço teórico deste trabalho. A Figura 1 ilustra quais critérios de inclusão e exclusão foram utilizados para filtrar as publicações. 
Figura 1 - Fluxograma da apuração dos artigos para sintetizar esta revisão.
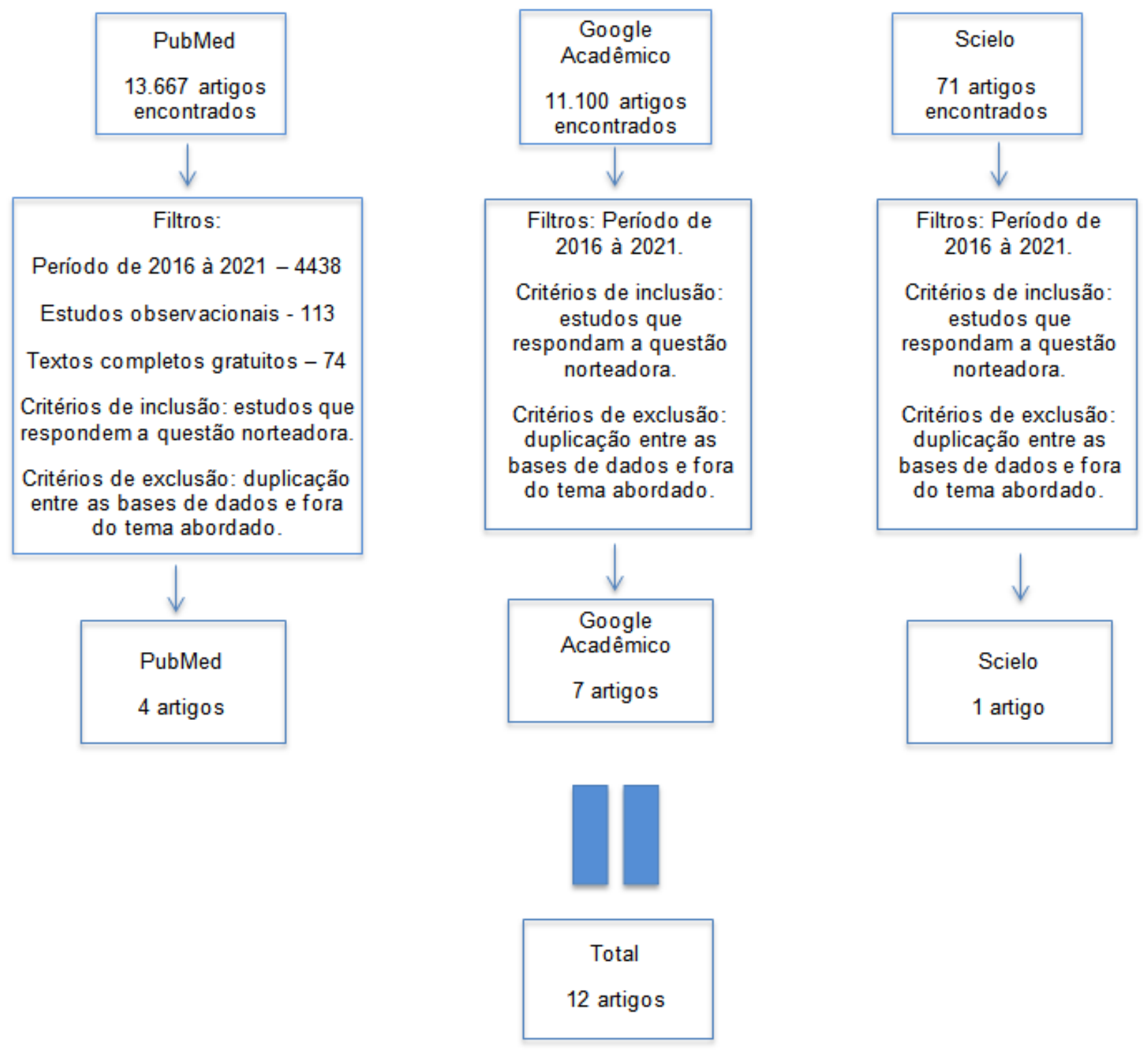

Fonte: BRUM LS, et al., 2021.

\section{RESULTADOS}

Primeiramente, partindo da seleção de 12 artigos com descritores específicos e verificados como pertinentes ao propósito desse trabalho, cada um deles foi arquivado para pesquisa, exploração e análise crítica. Por conseguinte, foi realizada uma leitura detalhada desses artigos selecionados e, além disso, eles também foram minuciosamente revisados e examinados, apurando-se os pontos mais relevantes no que diz respeito à temática abordada.

Tais artigos variam entre os anos de 2016 e 2021, a maioria deste último ano; sendo 8 nacionais, com destaque para região Sul, Sudeste e Nordeste do país, e 4 internacionais, com ênfase para estudos realizados na China e no Japão. São descritos como fatores de risco: a infecção pelo Helicobacter pylori (H. Pylori), fatores dietéticos, hábitos de vida (tabagismo, etilismo), baixa escolaridade, renda salarial, idade, IMC e predisposição genética. Em relação ao prognóstico, o estado nutricional do paciente, o estadiamento da lesão, a presença de comorbidades e a idade do paciente são relatados como os aspectos mais relevantes. 
A infecção pelo $H$. pylori representa o principal fator de risco ambiental para a ocorrência de câncer gástrico, principalmente o adenocarcinoma. Na população em geral, a infecção pelo $\mathrm{H}$. pylori atinge $60 \%$, mas em pacientes com câncer gástrico é mais comum (84\%). Sua presença eleva em cerca de seis vezes o risco desse tipo de tumor. É considerado carcinógeno tipo 1. Fatores como a virulência da cepa, suscetibilidade genética do hospedeiro (resposta imune do hospedeiro), duração da infecção, presença de inflamação crônica da mucosa e presença ou ausência de outros fatores ambientais, explicam porque a minoria dos pacientes infectados desenvolve a neoplasia. A maior virulência associada a predisposição ao adenocarcinoma ocorre nas cepas cagA+; cagE+ e vacA+. A participação da infecção pelo $H$. pylori é igualmente associada ao desenvolvimento da gastrite crônica superficial e da gastrite atrófica, passos iniciais na sequência da carcinogênese (MARTINS LC, et al., 2021).

A dieta tem sido descrita como um fator predisponente para o desenvolvimento do câncer gástrico. Alimentos ricos em sal ou conservados em sal, em especial carne seca salgada, peixes salgados, podem aumentar o risco da cronicidade da infecção pelo H.pylori, atuando portanto de forma sinérgica com a bactéria (MARTINS LC, et al., 2021).

O Índice de Massa Corporal (IMC) variando de 27,5 a 29,9 kg/m2 foi um fator de risco independente para displasia de alto grau na região da cárdia em ambos os sexos (HUANG Y, 2016). Vários estudos prospectivos e retrospectivos forneceram suporte para a hipótese de que IMC mais elevados em pacientes estão positivamente associados a uma maior incidência de câncer gástrico no cárdia. A obesidade associa-se a hiperinsulinemia e ao aumento do fator de crescimento semelhante à insulina 1, além de promover 0 desequilíbrio da adiponectina e leptina (HUANG Y, et al., 2016).

A abordagem cirúrgica convencional é uma das abordagens terapêuticas mais frequentes, entretanto relaciona-se ao aumento do catabolismo pós-operatório e a mudanças endócrinas, metabólicas, neuroendócrinas e imunitárias contribuindo para altas taxas de morbidade pós-operatória (POZIOMYCK AK, et al., 2017). Embora com decrescente incidência em muitos países nas últimas décadas, o câncer gástrico ainda apresenta distribuição mundial e prognóstico reservado (POZIOMYCK AK, et al., 2017).

Foi visto que as informações obtidas em todos os artigos selecionados apenas se agregam, demonstrando pouca divergência entre si, levando em conta os seguintes dados de cada periódico: fatores de risco, perfil epidemiológico analisado e prognóstico, demonstrados abaixo os dados dispostos no Quadro 1, para título de comparação e exemplificação do que foi dito. 
Quadro 1 - Resultados obtidos após análise detalhada dos periódicos.

\begin{tabular}{|c|c|c|c|c|}
\hline Título & $\begin{array}{l}\text { Autor/Ano de } \\
\text { publicação }\end{array}$ & Perfil Epidemiológico & Fatores de Risco & Prognóstico \\
\hline $\begin{array}{c}\text { Influência do regionalismo } \\
\text { Amazônico como fatore de risco } \\
\text { para o desenvolvimento de } \\
\text { câncer gástrico }\end{array}$ & $\begin{array}{l}\text { MARTINS LC, et al., } \\
2021\end{array}$ & $\begin{array}{l}32 \text { pacientes internados na clínico } \\
\text { cirúrgica do hospital Ophir Loyola. }\end{array}$ & $\begin{array}{c}\text { Idade maior que } 50 \text { anos, } \\
\text { baixa escolaridade e renda } \\
\text { salarial, consumo de sal em } \\
\text { alimentos processados, } \\
\text { farinha de mandioca e } \\
\text { infecção por Helicobacter } \\
\text { Pylori. }\end{array}$ & $\begin{array}{c}\text { Não foi encontrado no artigo o conteúdo } \\
\text { necessário. }\end{array}$ \\
\hline $\begin{array}{l}\text { Índice de massa corporal, } \\
\text { colesterol sérico e risco de } \\
\text { displasia gástrica de alto grau }\end{array}$ & $\begin{array}{l}\text { HUANG Y, et al., } \\
2016\end{array}$ & $\begin{array}{l}893 \text { pacientes com dispalasis } \\
\text { gástrica de alto grau e } 902 \\
\text { controles entre adultos chineses. }\end{array}$ & $\begin{array}{c}\text { IMC aumentado entre } \\
\text { homens e mulheres e o } \\
\text { colesterol total séricos em } \\
\text { mulheres. }\end{array}$ & $\begin{array}{c}\text { Não foi encontrado no artigo o conteúdo } \\
\text { necessário. }\end{array}$ \\
\hline $\begin{array}{c}\text { Xantoma gástrico é um marcador } \\
\text { preditivo para o início do câncer } \\
\text { gástrico detectado após } \\
\text { erradicação da Helicobacter } \\
\text { Pylori }\end{array}$ & $\begin{array}{l}\text { SCHIBUKAWA N, et } \\
\text { al., } 2019\end{array}$ & $\begin{array}{c}421 \text { pacientes que foram } \\
\text { submetidos à dissecção } \\
\text { endoscópica para câncer gástrico } \\
\text { precoce no hospital NTT West } \\
\text { Osaka. }\end{array}$ & $\begin{array}{c}\text { Xantoma gástrico } \\
\text { (xantelasmas ou ilhas de } \\
\text { lipídios). }\end{array}$ & $\begin{array}{c}\text { Não foi encontrado no artigo o conteúdo } \\
\text { necessário. }\end{array}$ \\
\hline $\begin{array}{l}\text { Predição de risco de câncer } \\
\text { gástrico: associação entre } \\
\text { ZBTB20 variante genética e risco } \\
\text { de câncer gástrico na população } \\
\text { chinesa Han }\end{array}$ & $\begin{array}{c}\text { BAI F e XIAO K } \\
2020\end{array}$ & $\begin{array}{l}509 \text { paciente com câncer gástrico } \\
\text { e } 507 \text { indivíduos saudáveis. }\end{array}$ & $\begin{array}{l}\text { Polimorfismo do nucleotídeo } \\
\text { único (SNPs) rs9841504 do } \\
\text { ZBTB20 em pacientes com } \\
\text { IMC > 24kg. }\end{array}$ & $\begin{array}{c}\text { Não foi encontrado no artigo o conteúdo } \\
\text { necessário. }\end{array}$ \\
\hline $\begin{array}{l}\text { Fatores de risco associados ao } \\
\text { desenvolvimento de câncer } \\
\text { gástrico - estudo caso-controle }\end{array}$ & $\begin{array}{l}\text { RAMOS MFKP, et } \\
\text { al., } 2017\end{array}$ & $\begin{array}{c}240 \text { casos de adenocarcinoma } \\
\text { gástrico, selecionados entre os } \\
\text { pacientes internados no Hospital } \\
\text { das Clínicas da Faculdade de } \\
\text { Medicina da Universidade de São } \\
\text { Paulo (HCFMUSP). }\end{array}$ & $\begin{array}{c}\text { Tabagismo e Etilismo, sem } \\
\text { predileção pelo tipo } \\
\text { histológico e localização da } \\
\text { lesão. }\end{array}$ & $\begin{array}{c}\text { Não foi encontrado no artigo o conteúdo } \\
\text { necessário. }\end{array}$ \\
\hline $\begin{array}{l}\text { Impacto do envelhecimento nos } \\
\text { resultados cirúrgicos de pacientes } \\
\text { com câncer gástrico }\end{array}$ & $\begin{array}{l}\text { DIAS AR, et al., } \\
2020\end{array}$ & $\begin{array}{c}600 \text { pacientes submetidos à } \\
\text { gastrectomia com intenção } \\
\text { curativa por adenocarcinoma } \\
\text { gástrico. }\end{array}$ & $\begin{array}{l}\text { Não foi encontrado no artigo } \\
\text { o conteúdo necessário. }\end{array}$ & $\begin{array}{c}\text { Pacientes com < } 55 \text { anos têm menos } \\
\text { complicações cirúrgicas. À medida que } \\
\text { idade avança, as complicações clínicas e } \\
\text { a mortalidade em } 90 \text { dias aumentam. A } \\
\text { gastrectomia subtotal é pior > } 72 \text { anos e } \\
\text { a linfadenectomia D2 deve ser } \\
\text { individualizada após essa idade. }\end{array}$ \\
\hline
\end{tabular}




\begin{tabular}{|c|c|c|c|c|}
\hline Título & $\begin{array}{l}\text { Autor/Ano de } \\
\text { publicação }\end{array}$ & Perfil Epidemiológico & Fatores de Risco & Prognóstico \\
\hline $\begin{array}{l}\text { Método de avaliação nutricional } \\
\text { preditores de mortalidade pós- } \\
\text { operátório em pacientes } \\
\text { submetidos à gastrectomia por } \\
\text { câncer gástrico }\end{array}$ & $\begin{array}{l}\text { POZIOMYCK AK, et } \\
\text { al., } 2017\end{array}$ & $\begin{array}{l}44 \text { pacientes portadores de } \\
\text { câncer gástrico. }\end{array}$ & $\begin{array}{l}\text { Não foi encontrado no artigo } \\
\text { o conteúdo necessário. }\end{array}$ & $\begin{array}{c}\text { A avaliação subjetiva global produzida } \\
\text { pelo paciente (ASG- PPP) e espessura } \\
\text { da mão dominante (MAPD) podem ser } \\
\text { utilizados como parâmetros pré- } \\
\text { operatórios para risco de morte após } \\
\text { gastrectomia. }\end{array}$ \\
\hline $\begin{array}{l}\text { Câncer gástrico em adultos } \\
\text { jovens: um grupo de pior } \\
\text { prognóstico? }\end{array}$ & $\begin{array}{l}\text { RAMOS MFKP, et } \\
\text { al., } 2019\end{array}$ & $\begin{array}{c}875 \text { pacientes, sendo } 84 \text { adultos } \\
\text { jovens e } 791 \text { com idade } \\
\text { avançada. }\end{array}$ & $\begin{array}{l}\text { Não foi encontrado no artigo } \\
\text { o conteúdo necessário. }\end{array}$ & $\begin{array}{l}\text { Jovens apresentaram uma menor } \\
\text { sobrevida livre da doença e a sobrevida } \\
\text { global foi semelhante entre os grupos e a } \\
\text { idade não mostrou ser um fator } \\
\text { prognóstico independente significativo. }\end{array}$ \\
\hline $\begin{array}{l}\text { Recorrência no câncer gástrico } \\
\text { PN0: fatores de risco no ocidente }\end{array}$ & $\begin{array}{l}\text { NOBRE KEL, et al., } \\
\qquad 2020\end{array}$ & $\begin{array}{l}270 \text { pacientes preencheram os } \\
\text { critérios de inclusão: } \\
\text { adenocarcinoma gástrico PNO, } \\
\text { gastrectomia com intenção } \\
\text { curativa e ressecção RO. }\end{array}$ & $\begin{array}{l}\text { Não foi encontrado no artigo } \\
\text { o conteúdo necessário. }\end{array}$ & $\begin{array}{l}\text { As recidivas aconteceram em pacientes } \\
\text { com }<16 \text { linfonodos ressecados, status } \\
\text { pt3-4, tumor } \geq 3,4 \mathrm{~cm} \text {, gastrectomia total } \\
\text { e presença de invasão linfática. }\end{array}$ \\
\hline $\begin{array}{l}\text { Relação entre estadiamento, } \\
\text { tratamento e sobrevida de } \\
\text { pacientes com câncer gástrico }\end{array}$ & $\begin{array}{l}\text { MENDES CP, et al., } \\
2021\end{array}$ & $\begin{array}{l}\text { Análise de } 135 \text { prontuários no } \\
\text { período de Janeiro de } 2015 \text { à } \\
\text { janeiro de } 2018 .\end{array}$ & $\begin{array}{l}\text { Não foi encontrado no artigo } \\
\text { o conteúdo necessário. }\end{array}$ & $\begin{array}{l}\text { Sobrevida geral do paciente submetido à } \\
\text { cirurgia por câncer gástrico foi de } 36,8 \\
\text { em } 5 \text { anos. }\end{array}$ \\
\hline $\begin{array}{l}\text { Sobrevivência e prognóstico de } \\
\text { adultos jovens com câncer } \\
\text { gástrico }\end{array}$ & $\begin{array}{l}\text { CORMEDI MVC, et } \\
\text { al., } 2018\end{array}$ & $\begin{array}{l}\text { Registros hospitalares de } 294 \\
\text { pacientes com câncer gástrico de } \\
\text { um hospital de referência em } \\
\text { câncer em São Paulo (SP). }\end{array}$ & $\begin{array}{l}\text { Não foi encontrado no artigo } \\
\text { o conteúdo necessário. }\end{array}$ & $\begin{array}{l}\text { A sobrevida geral foi semelhante entre } \\
\text { grupos de idade, a doença metastática } \\
\text { no diagnóstico foi o único fator associado } \\
\text { ao pior prognóstico em adultos jovens. }\end{array}$ \\
\hline $\begin{array}{l}\text { Levantamento e análise do } \\
\text { estado nutricional em pacientes } \\
\text { hospitalizados com tumores } \\
\text { gástricos malignos e sua } \\
\text { influência na qualidade de vida }\end{array}$ & $\begin{array}{l}\text { GUO ZQ, et al., } \\
2019\end{array}$ & $\begin{array}{l}2.322 \text { pacientes hospitalizados } \\
\text { com câncer gástrico. }\end{array}$ & $\begin{array}{l}\text { Não foi encontrado no artigo } \\
\text { o conteúdo necessário. }\end{array}$ & $\begin{array}{c}\text { Houve uma correlação negativa entre a } \\
\text { avaliação subjetiva global gerada pelo } \\
\text { paciente (PG-SGA) e vários parâmetros } \\
\text { nutricionais. A qualidade de vida de } \\
\text { pacientes com câncer gástrico é afetada } \\
\text { pela desnutrição. }\end{array}$ \\
\hline
\end{tabular}

REAC | Vol. 38 | DOI: https://doi.org/10.25248/REAC.e9214.2021

Página 7 de 10 


\section{DISCUSSÃO}

O câncer gástrico permanece como uma das mais importantes doenças malignas com diferenças geográficas, étnicas e socioeconômicas na sua distribuição. Seu desenvolvimento está associado a interação entre fatores ambientais, fatores ligados a bactéria e aqueles relacionados ao hospedeiro. Embora sua prevalência esteja em decrescimento, o câncer gástrico caracteriza-se como um importante problema de saúde pública (SOUZA LT, et al., 2017).

A bactéria Helicobacter pylori é reconhecida pela literatura como o principal fator ambiental associado ao desenvolvimento do adenocarcinoma (MARTINS LC, et al., 2021). A desproporção entre número de indivíduos infectados e aqueles que desenvolvem o câncer gástrico evidencia a necessidade de interação entre fatores da bactéria, suscetibilidade genética do indivíduo e fatores ambientais, como tabagismo e consumo de álcool (RAMOS MFKP, et al., 2017).

O papel da H. pylori na carcinogênese ainda não está completamente elucidado, entretanto é sabidamente promotor da gastrite crônica superficial, gastrite atrófica, metaplasia intestinal e displasia, consideradas lesões precursoras do adenocarcinoma (MARTINS LC, et al., 2021).

Estimam que o risco de câncer gástrico em indivíduos infectados é aumentado em 6 vezes (DIAS AA, et al., 2016). Conclui-se que sua erradicação trará efeitos positivos na prevenção do câncer gástrico principalmente nas populações de alto risco (RAMOS MFKP, et al., 2017).

Medidas preventivas para o câncer gástrico devem ser direcionadas para a conscientização e promoção de mudanças de hábitos de vida como: menor consumo de bebidas alcoólicas, evitar o tabagismo, dimunir o consumo de sal e alimentos conservados no sal; evitar o consumo exagerado de frituras, gorduras, carne vermelha, aflatoxina e compostos $\mathrm{N}$-nitrosos contidos em alimentos defumados, conservantes queijos e carnes curadas. Deve ser estimulada o consumo de uma dieta balanceada, composta por hortaliças cruas, frutas cítricas (ricas em ácido ascórbico (vitamina C) e betacaroteno, precursor da vitamina A). De igual importância é a promoção de melhorias nas condições sanitárias e socioeconômicas da população, conseguindo, assim, a diminuição da contaminação pelo $H$. pylori e da contaminação dos alimentos (SOUZA LT, et al., 2017).

Foi relatado que muitos tipos de câncer estão associados a um risco aumentado quando se tem a obesidade em questão. Vários estudos de análise prospectiva e retrospectiva forneceram suporte para a hipótese de que IMC mais elevados em pacientes estão positivamente relacionados a uma maior incidência de CG cardíaco. Ademais, os pacientes obesos têm sido associados ao desenvolvimento de muitos tipos de câncer, incluindo CG, pois costumam ter um alto nível de fator de crescimento semelhante à insulina 1. Além disso, estão relacionados a um risco aumentado devido ao acúmulo de tecido adiposo, induzindo desequilíbrios de adiponectina e leptina (HUANG T, et al., 2016).

Fatores como idade, estado nutricional e estadiamento do tumor no momento do diagnóstico influenciaram de forma negativa o prognóstico dos pacientes. A incidência de neoplasias malignas (incluindo GC) aumenta com o avanço da idade. Danos no DNA e exposição celular a carcinógenos se acumulam ao longo do tempo. Além disso, mudanças relacionadas à idade no reparo do DNA, sistemas imunológico e endócrino podem promover ou facilitar um conjunto de processos que levam a formação do câncer. Sendo assim, cresce a probabilidade da existência de várias doenças graves ou não, que contribuirão para um pior prognóstico (DIAS AR, et al., 2020).

Ainda, foi encontrado evidências de que alguns fatores de risco incomuns raramente podem influenciar no aparecimento de CG, como o Polimorfismo do Nucleotídeo Único (SNPs) ZBTB20 rs9288999 (BAI F e XIAO $\mathrm{K}, 2020)$. O Xantoma Gástrico também é considerado um fator raro para o aparecimento do CG (SCHIBUKAWA N, et al., 2019).

O estágio da doença é determinante para a escolha do tratamento mais adequado. A partir das opções de tratamento disponíveis para o câncer gástrico, em geral, a cirurgia é o meio mais utilizado. Sempre que possível, a ressecção completa do tumor permanece como tratamento padrão (SOUZA LT, et al., 2017). Mas 
se não for possível, deve ser priorizado a ressecção de mais de 16 linfonodos, a fim de evitar recidivas (NOBRE KEL, et al., 2020). Dependendo da localização e do estágio do tumor, gastrectomia parcial ou total pode ser necessária (SOUZA LT, et al., 2017).

Para melhorar ainda mais o controle local e regional do tumor e diminuir as taxas de metástases sistêmicas, estratégias de tratamento multimodal, incluindo quimioterapia e/ou radioterapia, podem ser utilizadas, pois foi constatado um pior prognóstico quando metástases estavam presentes no diagnóstico (CORMEDI MVC, et al., 2018).

É necessário uma avaliação nutricional pré-operatória mais individualizada para estes pacientes oncológicos possibilitando a identificação precoce de alterações nutricionais (POZIOMYCK AK, et al., 2017). Pacientes com câncer gástrico frequentemente apresentam emagrecimento, anorexia, fadiga, sensação de plenitude gástrica, vômitos, náuseas e desconforto abdominal, fatores estes que contribuem negativamente no decorrer da doença, aumentando as complicações, tempo de hospitalização e custos (SOUZA LT, et al., 2017).

Com isto, uma intervenção nutricional estabelecida previamente pode ser feita, visando à melhoria dos resultados pós-operatórios e à diminuição das taxas de mortalidade (POZIOMYCK AK, et al., 2017). É necessário ter atenção no momento do diagnóstico clínico, do tratamento e da triagem para a presença de pacientes desnutridos, sendo importante fornecer mudanças alimentares adequadas, visando 0 aumento a tolerância à terapia antitumoral e melhoria da qualidade de vida dos pacientes (GUO ZQ, et al., 2019).

As opções terapêuticas e a sobrevida dos pacientes acometidos pelo câncer gástrico estão diretamente relacionadas ao estadiamento no momento do diagnóstico e o início precoce do tratamento. A conscientização dos profissionais de saúde para ter-se bastante atenção em todas as etapas iniciais, a vigilância aos fatores de risco, e a investigação precoce do CG, possibilitará a mudança da morbimortalidade (MENDES CP, et al., 2021). Uma vez que o câncer gástrico é uma doença multifatorial, o pré-requisito para a prevenção é identificar e gerenciar com precisão os fatores de risco (BAI F e XIAO K, 2020).

\section{CONSIDERAÇÕES FINAIS}

Apresentando uma redução mundial na sua incidência, o câncer gástrico ainda se associa a grandes taxas de mortalidade. A identificação da infecção pelo Helicobacter pylori como principal fator de risco para o adenocarcinoma gástrico, assim como a participação de fatores dietéticos, demonstra a importância do desenvolvimento de políticas públicas que promovam a detecção e erradicação da infecção, bem como melhorias nas condições socioeconômicas da população, o que poderá impactar positivamente na redução da morbimortalidade. Ainda, deve ser levado em consideração a importância do diagnóstico precoce, do tratamento imediato e do acompanhamento de pacientes diagnósticados com câncer gástrico, contribuindo assim com a manutenção da qualidade de vida dos pacientes.

\section{REFERÊNCIAS}

1. BAI F, XIAO K. Prediction of gastric cancer risk: association between ZBTB20 genetic variance and gastric cancer risk in Chinese Han population. Bioscience reports, 2020; 40(9).

2. BESSA RA, et al. Apresentação clínica do câncer gástrico em paciente portador de HIV. Brazilian Journal of Health Review, 2019; 2(5): 4392-4399.

3. CORMEDI, MCV, et al. Survival and prognosis of young adults with gastric cancer. Clinics, 2018; 73.

4. DIAS AA, et al. Fatores de risco Genético e Ambientais para o câncer gástrico. Revista cientifica multidisciplinar Núcleo do conhecimento, 2016; 11(10): 63-72.

5. DIAS AC, et al. Impacto do envelhecimento nos resultados cirúrgicos dos pacientes com câncer gástrico. Arq. gastroenterol, 2021; 93-99.

6. GONÇALVES FV, et al. Perfil clínico epidemiológico do câncer gástrico: revisão integrativa. PubSaúde, 2020 ; 3; a041.

7. GUO ZQ, et al. Survey and analysis of the nutritional status in hospitalized patients with malignant gastric tumors and its influence on the quality of life. Supportive Care in Cancer, 2020; 28(1): 373-380. 
8. HUANG Y, et al. Body mass index, serum total cholesterol, and risk of gastric high-grade dysplasia: A case-control study among Chinese adults. Medicine, 2016; 95(35).

9. JÚNIOR AMF, et al. Câncer gástrico e fatores de risco ambientais: As influências do regionalismo amazônico e a infecção pela Helicobacter pylori. Revista Eletrônica Acervo Saúde, 2019; 11(13): e1115.

10. LOPES AC, et al. Associação entre obesidade e câncer gástrico. Revista Brasileira Militar de Ciências, $2020 ; 6$ (14).

11. MARTINS LC, et al. Influência do regionalismo amazônico como fator de risco para desenvolvimento de câncer gástrico. Enfermagem Brasil, 2021; 20(2): 130-142.

12. MENDES CP, et al. Relação entre estadiamento, tratamento e sobrevida de pacientes com câncer gástrico. Revista Eletrônica Acervo Saúde, 2021; 13(7): e8073.

13. NOBRE KEL, et al. Recorrência no Câncer Gástrico pNo: Fatores de Risco no Ocidente. Arquivos Brasileiros de Cirurgia Digestiva (ABDC), 2021; 34.

14. OLIVEIRA KSM, et al. Câncer de estômago: perfil epidemiológico de pacientes idosos. Revista Uningá Review, 2016; 28(1).

15. POZIOMYCK AK, et al. Métodos de avaliação nutricional preditores de mortalidade pós-operatória em pacientes submetidos à gastrectomia por câncer gástrico. Revista do Colégio Brasileiro de Cirurgiões, 2017; 44: 482-490.

16. RAMOS MFKP, et al. Câncer gástrico em adultos jovens: um grupo de pior prognóstico?. Revista do Colégio Brasileiro de Cirurgiões, 2019; 46.

17. RAMOS MFKP, et al. Fatores associados ao risco de desenvolvimento de adenocarcinoma gástrico: estudo casocontrole. Revista da Associação Médica Brasileira, 2017; 64 (7): 611-619.

18. SANTOS GRAC, et al. A situação do câncer gástrico em Salvador, no Brasil e no mundo. Revista Eletrônica Acervo Saúde, 2020; 53: e3629.

19. SILVA VCS, FELÍCIO DC. Fatores de risco para o câncer gástrico em grupos de classe socioeconômico baixa: revisão literária. Revista de Iniciação Científica da Universidade Vale do Rio Verde, 2016; 6(1).

20. SOUZA LT, et al. Implicações nutricionais no câncer gástrico: uma revisão. Journal of Applied Pharmaceutical Sciences (JAPHAC), 2017; 4(3): 2-13.

21. SHIBUKAWA N, et al. Gastric xanthoma is a predictive marker for early gastric cancer detected after helicobacter pylori eradication. Internal Medicine, 2019, 58(6): 779-7840.

22. ZILBERSTEIN B, et al. Consenso brasileiro sobre câncer gástrico: diretrizes para o câncer gástrico no Brasil. Arquivos Brasileiros de Cirurgia Digestiva (ABCD), 2013; 26; 2-6. 